\title{
ORIGINAL
}

\section{ASOCIACIONES ENTRE LA RED SOCIAL Y LA DISCAPACIDAD AL COMIENZO DE LA VEJEZ EN LAS CIUDADES DE MADRID Y BARCELONA EN 2005 (*)}

\author{
Miguel Ángel Escobar Bravo (1), Dolores Puga (2) y Montserrat Martín (3) \\ (1) Universidad de Lleida. \\ (2) Consejo Superior de Investigaciones Científicas. \\ (3) Universidad Autónoma de Barcelona.
}

\section{RESUMEN}

Fundamento: La pérdida de autonomía a edades avanzadas no se asocia únicamente con el envejecimiento sino también con características del entorno físico y social. Investigaciones recientes han demostrado que la red social, la integración social y la participación, actúan como predictores de la discapacidad en la vejez. El objetivo de este trabajo es analizar el efecto de la red social sobre el nivel de autonomía (en términos de discapacidad instrumental y básica) en etapas iniciales de la vejez.

Métodos: La fuente de datos fue la primera oleada de la encuesta "Procesos de Vulnerabilidad en la Vejez", realizada en 2005 a una muestra de 1.250 individuos que, tras la depuración posterior de los datos, resultó en un tamaño muestral final de 1.244 individuos. La población objeto de estudio fue la cohorte entre 70 y 74 años residente en grandes áreas urbanas (Madrid y Barcelona) y no institucionalizada. Se midió discapacidad para el desarrollo de actividades básicas de la vida diaria (ABVD), y actividades instrumentales de la vida diaria (AIVD). Los aspectos estructurales de las relaciones sociales se midieron a través de la diversidad de red social y la participación, para lo que se construyó un indicador global de diversidad de red social

Resultados: Por cada unidad que disminuye el indicador de diversidad de red, la probabilidad de padecer una discapacidad instrumental frente a no padecer ningún tipo de discapacidad, se multiplica por 0,97 (IC95\% 0,95-0,99), y la probabilidad de padecer una discapacidad básica se multiplica por 0,91 (IC95\% 0,87-0,94)

Conclusiones: Al igual que en otros ámbitos geográficos, existe una relación estadísticamente significativa entre el indicador de diversidad de red social utilizado y la discapacidad en sus dos niveles de gravedad. Por tanto, la diversidad de la red social parece mostrar un efecto protector frente a la discapacidad entre la población urbana al comienzo de la vejez.

Palabras clave: Envejecimiento. Relaciones sociales. Redes sociales. Discapacidad. Envejecimiento saludable.

Correspondencia:

Miguel Ángel Escobar Bravo

Rda. Zamenhof $1376^{\circ} 2^{\mathrm{a}}$

08202 Sabadell

maescobar@telefonica.net

ABSTRACT

\section{Protective effects of social networks on disability among older adults in Madrid and Barcelona, Spain, in 2005}

Background: The loss of autonomy at advanced ages is not only associated with ageing, but also with the characteristics of the physical and social environment. Recent investigations have shown that social networks, social engagement and participation act like predictors of disability among the elderly. To analyze the effect of social networks on the level of autonomy (instrumental and basic disability) at initial stages of the old age.

Methods: The source of data is the first wave of the survey "Processes of Vulnerability among Spanish Elderly", carried out in 2005 to a sample of 1,250 individuals? with the later purification of the data, it was in a size final sample of 1.244 individuals? The population object of study is the cohort aged 70 to 74 years in metropolitan areas (Madrid and Barcelona) and not institutionalized. Disability is measured by the development of basic activities of daily life (ADL), and instrumental activities of daily life (IADL). The structural aspects of the social relationships are measured through the diversity of social networks and participation, for which a global indicator of social network diversity was built.

Results: For each unit that diminishes the indicator of social network diversity, the probability of suffering an instrumental disability ?in front of not suffering any disability? is multiplied by 0.97 (IC95\% $0.95-0.99$ ), and the probability of suffering a basic disability is multiplied by 0.91 (IC95\% $0.87-0.94$ )

Discussion: Like in other geographic scopes, a statistically significant relationship exists between the indicator of diversity of social network used and the disability, in its two levels of graveness. Therefore, the diversity of the social network seems to show a protective effect in front of the disability among the Spanish urban population at the beginning of the age.

Key words: Aging. Social support. Disabled Persons. Health Status Disparities.

(*) Proyecto financiado por el Fondo de Investigación Sanitaria (No exp 04/1948-2). 


\section{INTRODUCCIÓN}

Si bien la discapacidad no es una consecuencia inevitable del envejecimiento, las situaciones de fragilidad son crecientes a edades avanzadas, pudiendo desembocar en pérdida de autonomía y dependencia ${ }^{1-5}$. El deterioro funcional y cognitivo que se puede alcanzar en momentos avanzados de la vejez no se asocia tan sólo con el envejecimiento, sino también con el estilo de vida y las características del entorno del individuo. Investigaciones recientes han puesto de manifiesto que más allá de las condiciones biológicas específicas, los factores externos, sociales y ambientales, explican las variaciones en las formas de envejecer ${ }^{6-12}$.

A factores de riesgo conocidos como el nivel educativo, el nivel de ingresos, el estrato social, o los hábitos de vida ${ }^{6-}$ $8,11,15-19,24$ se unen factores tales como la red social próxima del mayor, su integración social y participación, que afectan a la vulnerabilidad social del individuo, actuando como predictores de situaciones personales relativas a la salud, la autonomía funcional y cognitiva, el bienestar y la satisfacción entre las personas de edad $^{1,13-23,25}$.

La influencia protectora de las relaciones sociales sobre la salud ha sido objeto de diversas investigaciones recientes ${ }^{12,26-28}$. Diferentes autores han demostrado que los mayores que disfrutan de amplias relaciones sociales tienen mayores probabilidades de sobrevivir ${ }^{29,30}$, y muestran mejores resultados en salud y autonomía ${ }^{31}$. La amplitud de las redes sociales, la frecuencia del contacto con amigos, o la fortaleza de la red familiar $26,27,32$, han mostrado efectos protectores frente al inicio de discapacidad, frenando su progresión e incluso incrementando el grado de recuperación.

La discapacidad es un fenómeno complejo, que resulta de la relación entre la salud individual, el entorno, las características personales y los factores psicosociales. El modelo de discapacidad propuesto por Verbrugge y Jette ${ }^{33}$, a partir del modelo inicial de $\mathrm{Nagi}^{35-37}$, es el más utilizado y el más ampliamente aceptado en la investigación sobre discapacidad, gracias a su precisión y flexibilidad, mostrando una gran capacidad de adaptación a diversos enfoques. Dicho modelo define el proceso de discapacidad como la dificultad para realizar actividades que se consideran habituales entre individuos de las mismas características en el mismo entorno físico y social, vinculándola en mayor medida a la función social que a la función orgánica, a la que se asocian patología y deficiencia ${ }^{41}$. A menudo, este concepto se hace operativo en términos de dificultad o incapacidad para la realización de actividades básicas de la vida diaria (ABVD), y actividades instrumentales de la vida diaria (AIVD) ${ }^{40}$.

El concepto de relaciones sociales hace referencia al conjunto de personas con las que interacciona el individuo, a las características de los vínculos que se establecen entre ellas, y a las interacciones que se producen a través de dichos vínculos. Durante la última década la mayor parte de los autores, de forma explícita ${ }^{42,43}$, o implícita en los análisis desarrollados ${ }^{12,20,22,23,26,28,32,44}$, han coincidido en la necesidad de diferenciar entre la estructura de las relaciones sociales y su función. Así, la red social hace referencia a los aspectos estructurales de las relaciones sociales, comprendiendo el tejido de individuos con los que uno tienen relaciones interpersonales y las características de los vínculos que se establecen -en términos de número de miembros, frecuencia de contactos, diversidad de vínculos, reciprocidad o intimidad-. La integración social de los mayores se produce mediante redes informales (aquellas que comprenden los vínculos con familiares y amigos), y redes forma- 
les o participación. La composición de la red social, con una mayor presencia de vínculos informales, o una mayor participación formal, se ve fuertemente influenciada por condicionantes culturales y políticos de la sociedad de pertenencia $^{12,22,26,42,45,46}$. La función de las relaciones sociales, o apoyo social, hace referencia a las interacciones personales dentro de dicha estructura, al contenido relacional de las mismas -apoyo instrumental, emocional, vinculación social o desarrollo de roles sociales, influencia social, e incluso la tensión social o efectos negativos de las relaciones sociales-. El flujo de apoyo social opera sobre el bienestar a través de la estructura de la red social.

Resultados de investigaciones previas han demostrado que no todos los componentes de las relaciones sociales son igualmente beneficiosos ${ }^{26}$, las asociaciones son complejas, de forma que los efectos de la red y el apoyo social sobre la salud son diferentes ${ }^{12}$. Las medidas que enfatizan en los componentes estructurales de las relaciones sociales muestran un efecto protector frente a la discapaci$\operatorname{dad}^{12,23,28,32,44}$, e incluso un mayor efecto protector frente a la progresión de la discapacidad a largo plazo que aquellos que enfatizan en aspectos funcionales ${ }^{26}$. La red social ha sido identificada como un factor protector frente al declive funcio$\mathrm{nal}^{44}$, al inicio de discapacidad bási$\mathrm{ca}^{12,26,32}$, y asociada con una mayor probabilidad de recuperación tras la misma $^{12,32}$. Sin embargo la evidencia no es concluyente, mientras los resultados del Alameda County Study ${ }^{47}$, del Established populations for epidemiological studies od the elderly (EPESE) ${ }^{20,26}$, del Nordic Research on Aging (NORA) ${ }^{28}$ o del Comparision of Longitudinal European Studies on Aging (CLESA) ${ }^{12}$ han mostrado fuertes relaciones entre red social y discapacidad, los datos del MacArthur Study of Successful Aging no muestra dichos efectos protectores ${ }^{46,48}$. No obstante, algunas investigaciones se vieron obstaculizadas por el uso de indicadores brutos y no específicos de redes sociales que, en su mayor parte, no diferenciaban entre diversos tipos de vínculos.

El objetivo de este estudio es analizar la asociación entre la red social y la autonomía (en términos de discapacidad básica e instrumental) al comienzo del período de mayor riesgo de entrada en discapacidad.

\section{SUJETOS Y MÉTODOS}

La fuente de datos es la primera oleada de la encuesta "Procesos de Vulnerabilidad en la Vejez", realizada mediante entrevista personal domiciliar, en primavera de 2005 , a una muestra de 1.250 individuos. La población objeto de estudio está constituida por la cohorte entre 70 y 74 años residente en grandes áreas urbanas (áreas metropolitanas de Madrid y Barcelona) y en viviendas familiares (no institucionalizados).

\section{Variables e instrumentos de medida}

Discapacidad: Se mide discapacidad mediante el auto-reporte de dificultades y/o incapacidad para el desarrollo de actividades básicas de la vida diaria (ABVD), y actividades instrumentales de la vida diaria (AIVD). Las actividades analizadas son las observadas en los test de $\mathrm{Katz}^{50}$ y Lawton ${ }^{51}$ respectivamente.

En este análisis se utiliza una variable multinomial: 1) sin discapacidad; 2) sólo con discapacidad instrumental (AIVD); y 3) con alguna discapacidad básica (ABVD). Con esta categorización se pretende clasificar a los sujetos según el diferente nivel de atención y cuidados que necesitan. 
Red social y participación social: Los aspectos estructurales de las relaciones sociales se miden a través de la diversidad de red social y de la participación. Al igual que otros estudios ${ }^{12,52}$, en primer lugar se realizó un análisis factorial confirmatorio que permitió definir los dominios que organizan los vínculos sociales en términos rol-específico: vínculos dentro del hogar, con hijos y nietos, con hermanos y amigos, intimidad, asociacionismo, actividades sociales, y dinamismo. Las características de la red social (número de miembros, proximidad, frecuencia de contactos) se miden de forma diferenciada para cada uno de los vínculos. La suma de las puntuaciones parciales permite obtener un indicador global de diversidad de red (presencia y fortaleza de contactos en distintos tipos de vínculos). Las medidas de frecuencia de contactos se observan para contactos personales y telefónicos, la importancia de observar también estos últimos es resaltada por Avlund y colegas, puesto que su contenido puede ser complementario ${ }^{28}$ :

1. Índice de red social: con un rango entre 0 y 22 puntos, mide la diversidad de los componentes de la red social, la proximidad con los distintos vínculos y la frecuencia de contactos, así como intimidad (presencia de confidente): a) hogar: presencia de pareja; tamaño $(0,1,2,3$ o más); b) hijos y nietos: tamaño de la red $(0,1,2,3,4$ o más); distancia a la que viven (entre 0 y 15 minutos, entre 15 y $30 \mathrm{~min}$., o más de 30 min.) y frecuencia de contacto (se ven diariamente; se ven semanalmente o hablan por teléfono diariamente; se ven mensualmente o hablan por teléfono semanalmente; se ven menos de mensualmente o hablan por teléfono mensualmente o menos); c) hermanos y amigos: tamaño de la red (0,1,2,3,4 o más); distancia a la que viven (entre 0 y 30 minutos, o más de 30 min.) y frecuencia de contacto (se ven diariamente; se ven semanalmente o hablan por teléfono dia- riamente; se ven mensualmente o menos o hablan por teléfono semanalmente o menos); y d) confidente: presencia; y frecuencia de contacto (se ven a diario, semanalmente o menos).

2. Índice de participación social: Rango entre 0 y 23 puntos y constituido por: a) asociacionismo: pertenencia a alguna asociación; b) actividades sociales: frecuencia con que acude al mercado o tiendas; da paseos por la plaza o el parque del barrio; acude al hogar o club del pensionista; acude a la iglesia o centro de culto (nunca, diariamente, semanalmente o mensualmente); y c) dinamismo: comienzo o abandono de alguna actividad nueva recientemente.

En la tabla 2 se muestra la distribución de frecuencias en la muestra de los componentes tanto de diversidad social como de participación que contribuyen al indicador de red social.

El indicador construido, con rango entre 0 y 45 puntos, presenta una coherencia interna aceptable (alfa de Cronbach de 0,66 para el indicador completo, de 0,70 para la estructura de la red y de 0,59 para la participación social).

Variables de control: En el análisis se introduce información relativa a variables que pueden actuar como confusores, con el objeto de controlar su efecto. Se trata de variables demográficas y socio-económicas básicas, como edad, género, nivel de ingresos de hogar, tenencia de la vivienda, nivel educativo completado, comorbilidad (número de enfermedades diagnosticadas) y la ocurrencia de eventos biográficos negativos en los últimos 10 años (fallecimiento de algún familiar cercano, enfermedad grave de algún familiar cercano, fallecimiento de un amigo, jubilación propia o del cónyuge, disminución de los ingresos del hogar). 
Análisis de los datos: Para el análisis se han utilizado técnicas de regresión logística multinominal. En un primer paso seleccionamos las variables que se introducirían en el modelo multivariante mediante análisis bivariados, siguiendo las recomendaciones de Hosmer y Lemeshow ${ }^{53}$. Los test estadísticos que se utilizaron fueron, según la naturaleza de las variables, ANOVA o chi cuadrado o sus equivalentes en los casos que no se cumplieran los criterios de aplicación de estos test. Toda variable independiente que obtuvo un grado de significación inferior a 0,25 en estos análisis fue introducida en el modelo. Posteriormente se realizaron los análisis definitivos utilizando regresión logística multinominal.

\section{RESULTADOS}

La media de edad de la muestra fue de 72,7 (DE 1,7) años, representando las mujeres el 57\% de la muestra (Tabla 1). La

Tabla 1

Distribución de las características sociodemográficas de los sujetos de estudio

\begin{tabular}{|c|c|c|}
\hline Variable & $\mathbf{n}$ & $\%$ \\
\hline Edad & 1.171 & $72,7(1,7)^{*}$ \\
\hline Sexo & 1.244 & \\
\hline Hombre & 531 & 42,7 \\
\hline Mujer & 713 & 57,3 \\
\hline Ingresos & 1.235 & \\
\hline $600 €$ o menos & 294 & 23,6 \\
\hline Más de $600 €$ & 588 & 47,3 \\
\hline Prefiere no contestar & 353 & 28,4 \\
\hline Vivienda & 1.240 & \\
\hline Propietario & 964 & 77,7 \\
\hline No propietario & 276 & 22,3 \\
\hline Estudios & 1.228 & \\
\hline Primarios o menos & 762 & 62,1 \\
\hline Secundarios o más & 466 & 37,9 \\
\hline Eventos & 1.244 & \\
\hline Ninguno & 101 & 8,1 \\
\hline 1, 263 & 823 & 66,2 \\
\hline 4 o más & 320 & 25,7 \\
\hline Comorbilidad & 1.244 & $2,3(1,6)^{*}$ \\
\hline
\end{tabular}

mayor parte de la población tiene un nivel de ingresos superior a los $600 €$ mensuales -si bien un tercio de los encuestados prefirieron no declarar su nivel de ingresos- y son propietarios de la vivienda en la que residen. El $38 \%$ ha realizado estudios secundarios o superiores, cifra que difiere notablemente $(15,6 \%)$ de la presentada en el Informe 2004 del IMSERSO (55); esta diferencia se explica por la composición urbana, y en edades iniciales de la vejez (70-74 años) de la cohorte objeto de estudio, mientras que en el informe mencionado se hace referencia al conjunto de población española de 65 y más años. Solamente el $8 \%$ de los encuestados no ha vivido un evento biográfico negativo en los últimos tiempos y presentan una media de 2,3 (DE 1,6) de enfermedades crónicas diagnosticadas.

Red social: En la tabla 2 se muestra la descripción pormenorizada de la red social. La mayoría de personas tienen pareja, pero un $23 \%$ de los sujetos vive solo. Cerca del $73 \%$ de esta población tiene dos o más hijos vivos de los que el $40 \%$ vive muy cerca de ellos (menos de 15 minutos) y con los que mantienen un contacto al menos semanalmente casi el $70 \%$. En cuanto a los nietos, el $33 \%$ de las personas encuestadas tiene cuatro o más, de los que algo más de la mitad viven a menos de 30 minutos de sus casas y tienen contacto al menos semanalmente con ellos el 57\%. Por otro lado, casi el $60 \%$ tienen dos o más hermanos vivos que viven, en su mayoría (57\%) a más de media hora de distancia, y con los que mayoritariamente $(69 \%)$ tienen contacto semanalmente o con mayor frecuencia. Por lo que hace referencia a los amigos, la mitad de las personas encuestadas dicen tener 4 o más, y un $69 \%$ viven cerca de ellos (menos de 30 minutos), pero en un $40 \%$ de los casos tienen contacto con ellos con una frecuencia inferior a la semanal. Un $64 \%$ dicen contar con alguna persona de confianza a la que, en un $42 \%$ de los casos, ven diariamente. 
Tabla 2

Frecuencias de las variables que componen el indicador de red social

\begin{tabular}{|c|c|c|c|c|c|c|c|c|}
\hline \multicolumn{6}{|c|}{ Diversidad social } & \multicolumn{3}{|l|}{ Participación social } \\
\hline Variable & $\mathbf{n}$ & $\%$ & Variable & $\mathbf{n}$ & $\%$ & Variable & $\mathbf{n}$ & $\%$ \\
\hline Pareja & & & Hermanos & & & Asociacionismo & & \\
\hline $\mathrm{Si}$ & 797 & 64,1 & Ninguno & 196 & 15,8 & No & 934 & 76,1 \\
\hline No & 447 & 35,9 & Uno & 304 & 24,4 & Sí & 293 & 23,9 \\
\hline Tamaño del hogar & & & Dos o tres & 458 & 36,8 & Mercado, compras & & \\
\hline Vive solo & 291 & 23,4 & Cuatro o más & 267 & 21,5 & Nunca & 148 & 11,9 \\
\hline Vive con otra persona & 630 & 50,6 & Distancia hermanos & & & $<4$ veces/mes & 95 & 7,6 \\
\hline Vive con dos personas & 220 & 17,7 & Más de $30 \mathrm{~m}$ & 711 & 57,2 & Una vez a la semana & 161 & 12,9 \\
\hline Vive con tres o más & 103 & 8,3 & Entre 30 y $0 \mathrm{~m}$ & 533 & 42,8 & Entre 5 y 29 veces $/ \mathrm{mes}$ & 655 & 52,7 \\
\hline Hijos & & & Contacto hermanos & & & 30 ó más veces/mes & 185 & 14,9 \\
\hline Ninguno & 160 & 13,0 & Menos de semanalmente & 860 & 69,1 & Plaza, parque & & \\
\hline Uno & 178 & 14,5 & Semanalmente o tlf. diario & 250 & 20,1 & Nunca & 210 & 16,9 \\
\hline Dos o tres & 694 & 56,4 & Diariamente & 134 & 10,8 & $<4$ veces/mes & 70 & 5,6 \\
\hline Cuatro o más & 199 & 16,2 & Amigos & & & Una vez a la semana & 79 & 6,4 \\
\hline Distancia hijos & 911 & & Ninguno & 243 & 20,1 & Entre 5 y 29 veces/mes & 437 & 35,1 \\
\hline Más de $30 \mathrm{~m}$ & 341 & 37,4 & Uno & 102 & 8,4 & 30 ó más veces/mes & 448 & 36,0 \\
\hline Entre 30 y $15 \mathrm{~m}$ & 205 & 22,5 & Dos o tres & 250 & 20,7 & Hogar, club pensionista & & \\
\hline Menos de $15 \mathrm{~m}$ & 365 & 40,1 & Cuatro o más & 614 & 50,8 & Nunca & 994 & 79,9 \\
\hline Contacto hijos & 911 & & Distancia amigo & & & $<4$ veces/mes & 72 & 5,8 \\
\hline Menos de mensual o tlf. mensual & 220 & 24,1 & Más de $30 \mathrm{~m}$ & 383 & 30,8 & Una vez a la semana & 58 & 4,7 \\
\hline Mensualmente o tlf. semanal & 60 & 6,6 & Entre 30 y $0 \mathrm{~m}$ & 861 & 69,2 & Entre 5 y 29 veces/mes & 91 & 7,3 \\
\hline Semanalmente o tlf. diario & 354 & 38,9 & Contacto amigos & & & 30 ó más veces/mes & 29 & 2,3 \\
\hline Se ven a diario & 277 & 30,4 & Menos de semanalmente & 504 & 40,5 & Centro de culto & & \\
\hline Nietos & & & Semanalmente o tlf. diario & 379 & 30,5 & Nunca & 560 & 45,0 \\
\hline Ninguno & 289 & 24,0 & Diariamente & 361 & 29,0 & $<4$ veces/mes & 210 & 16,9 \\
\hline Uno & 174 & 14,5 & Confidente & & & Una vez a la semana & 320 & 25,7 \\
\hline Dos o tres & 347 & 28,8 & No & 450 & 36,2 & Entre 5 y 29 veces/mes & 117 & 9,4 \\
\hline Cuatro o más & 394 & 32,7 & Sí & 794 & 63,8 & 30 ó más veces/mes & 37 & 3,0 \\
\hline Distancia nietos & 118 & 4 & Contacto confidente & & & Inicio actividad nueva & & \\
\hline Más de $30 \mathrm{~m}$ & 565 & 47,7 & Menos de semanalmente & 492 & 39,5 & No & 989 & 79,5 \\
\hline Entre 30 y $15 \mathrm{~m}$ & 212 & 17,9 & Semanalmente & 224 & 18,0 & Sí & 252 & 20,3 \\
\hline Menos de $15 \mathrm{~m}$ & 407 & 34,4 & Diariamente & 528 & 42,4 & Abandono actividad & & \\
\hline Contacto nietos & 1.184 & & & & & No & 888 & 71,7 \\
\hline Menos de mensual o tlf. mensual & 407 & 34,4 & & & & Sí & 351 & 28,3 \\
\hline Mensualmente o tlf. semanal & 106 & 9,0 & & & & & & \\
\hline Semanalmente o tlf. diario & 398 & 33,6 & & & & & & \\
\hline Se ven a diario & 273 & 23,1 & & & & & & \\
\hline
\end{tabular}

En cuanto a la participación social (tabla 2) una gran mayoría de la población (76\%) observada no forma parte de ninguna asociación o club, alrededor del $70 \%$ va a hacer compras $(68 \%)$ y a pasear por el barrio $(71 \%)$ más de una vez a la semana; muy pocos $(20 \%)$ acude al menos una vez al mes a un centro de la tercera edad y un $45 \%$ no va nunca a un centro de culto. Por otro lado, casi el $80 \%$ no ha iniciado ninguna actividad nueva en los últimos años y un $28 \%$ ha abandonado alguna actividad que venía realizando.

La puntuación media del indicador de red social es de 20,4 sobre un máximo de 45 , siendo significativamente superior entre la población con un mayor nivel 
socio-económico (Tabla 3): población con mayores ingresos $(\mathrm{p}=0,002)$ y propietarios de la vivienda ( $p<0,0005)$. Los eventos biográficos negativos parecen hacer fortalecer la red social de forma significativa $(\mathrm{p}=0,008)$. También se puede observar que conforme aumenta la edad de las personas encuestadas la puntuación media en el indicador de red social es menor $(\mathrm{p}=0,001)$.

En cuanto al índice de diversidad social, la puntuación media es de 11,5 sobre un máximo de 22, siendo significativamente superior en los hombres ( $\mathrm{p}=0,026)$, en los que tienen más de $600 €$ de ingresos mensuales del hogar $(\mathrm{p}=0,001)$, en los propietarios de la vivienda ( $\mathrm{p}<0,0005)$, en los que han cursado estudios primarios o menos $(\mathrm{p}=0,025)$ y disminuye según aumenta la edad de los encuestados ( $\mathrm{p}=$ 0,001). Por lo que se refiere al índice de participación social, la puntuación media obtenida es de 8,8 sobre un máximo de 23 .
En este apartado, las diferencias estadísticamente significativas encontradas son: obtienen una puntuación superior las personas con más ingresos económicos $(\mathrm{p}=0,049)$ y propietarios de su vivienda ( $\mathrm{p}<0,0005)$; los que han cursado estudios secundarios o más $(\mathrm{p}=0,044)$ y aquellos que han sufrido más eventos negativos $(\mathrm{p}=0,005)$.

El indicador de red social global muestra también una distribución significativa según el nivel de discapacidad $(\mathrm{p}<0,0005)$ : a menor fortaleza y diversidad de la red social, mayor gravedad de la discapacidad (tabla 4). Lo mismo ocurre con los índices de diversidad social $(\mathrm{p}=0,009)$ y de participación social $(\mathrm{p}<0,0005)$.

Discapacidad según variables sociodemográficas básicas: La prevalencia de discapacidad instrumental masculina (Tabla 5) es sensiblemente superior a la femenina $(30,1$ versus 18,$2 ; \mathrm{p}<0,0005)$, debido a la mayor presencia de problemas

Tabla 3

Comparación de medias del indicador de red social según las variables de control.

\begin{tabular}{|c|c|c|c|c|c|c|c|c|c|c|}
\hline & \multirow{2}{*}{$\mathbf{n}$} & \multicolumn{3}{|c|}{ Indicador de red social } & \multicolumn{3}{|c|}{ Índice de diversidad social } & \multicolumn{3}{|c|}{ Índice de participación social } \\
\hline & & Media & $\mathrm{DE}^{*}$ & $\mathbf{p}$ & Media & $\mathrm{DE}^{*}$ & $\mathbf{p}$ & Media & $\mathrm{DE}^{*}$ & $\mathbf{p}$ \\
\hline Total población & 1.244 & 20,4 & 5,9 & & 11,5 & 3,7 & & 8,8 & 3,9 & \\
\hline \multicolumn{11}{|l|}{ Sexo } \\
\hline Hombre & 492 & 20,6 & 6,0 & 0,245 & 11,8 & 3,7 & 0,026 & 8,8 & 4,0 & 0,899 \\
\hline Mujer & 652 & 20,2 & 5,8 & & 11,3 & 3,7 & & 8,8 & 4,0 & \\
\hline \multicolumn{11}{|l|}{ Ingresos } \\
\hline $600 €$ o menos & 274 & 19,4 & 5,7 & 0,002 & 10,9 & 3,8 & 0,001 & 8,5 & 3,6 & 0,049 \\
\hline Más de $600 €$ & 864 & 20,7 & 5,9 & & 11,7 & 3,7 & & 9,0 & 4,0 & \\
\hline \multicolumn{11}{|l|}{ Vivienda } \\
\hline Propietario & 893 & 21,2 & 5,7 & $<0,0005$ & 12,0 & 3,6 & $<0,0005$ & 9,2 & 3,9 & $<0,0005$ \\
\hline No propietario & 248 & 17,4 & 5,8 & & 9,8 & 3,8 & & 7,7 & 3,8 & \\
\hline \multicolumn{11}{|l|}{ Estudios } \\
\hline Primarios o menos & 701 & 20,4 & 5,8 & 0.862 & 11,7 & 3,7 & 0,025 & 8,6 & 3,9 & 0,044 \\
\hline Secundarios o más & 430 & 20,5 & 6,0 & & 11,2 & 3,7 & & 9,1 & 4,0 & \\
\hline \multicolumn{11}{|l|}{ Eventos } \\
\hline Ninguno & 96 & 19,0 & 5,1 & 0,008 & 11,0 & 3,8 & 0,158 & 7,8 & 3,2 & 0,005 \\
\hline 1,263 & 759 & 20,3 & 6,0 & & 11,5 & 3,8 & & 8,8 & 4,0 & \\
\hline 4 o más & 289 & 21,1 & 5,8 & & 11,8 & 3,4 & & 9,3 & 4,0 & \\
\hline Comorbilidad** & 1.244 & \multicolumn{2}{|c|}{$\mathrm{R}=-0,039$} & 0,188 & \multicolumn{2}{|c|}{$R=-0,012$} & 0,685 & \multicolumn{2}{|c|}{$\mathrm{R}=0,041$} & 0,151 \\
\hline Edad** & 1.171 & \multicolumn{2}{|c|}{$\mathrm{R}=-0,097$} & 0,001 & \multicolumn{2}{|c|}{$R=-0,104$} & 0,001 & \multicolumn{2}{|c|}{$\mathrm{R}=-0,043$} & 0,149 \\
\hline
\end{tabular}

* Desviación estándar.

* Para la edad y la comorbilidad se aplica la correlación de pearson. 
Tabla 4

Comparación de medias del indicador de red social según el nivel de discapacidad

\begin{tabular}{|c|c|c|c|c|c|c|c|c|c|c|}
\hline & \multirow{2}{*}{$\mathbf{n}$} & \multicolumn{3}{|c|}{ Indicador de red social } & \multicolumn{3}{|c|}{ Índice de diversidad social } & \multicolumn{3}{|c|}{ Índice de participación social } \\
\hline & & Media & DE* $^{*}$ & $\mathbf{p}$ & Media & DE* & $\mathbf{p}$ & Media & DE* & $\mathbf{p}$ \\
\hline Total población & 1.244 & 20,4 & 5,9 & & 11,5 & 3,7 & & 8,8 & 3,9 & \\
\hline Sin discapacidad & 730 & 21,0 & 5,7 & \multirow{3}{*}{$<0,0005$} & 11,6 & 3,8 & \multirow{3}{*}{0,009} & 9,3 & 3,7 & \multirow{3}{*}{$<0,0005$} \\
\hline Discapacidad instrumental & 265 & 20,5 & 5,5 & & 11,6 & 3,6 & & 8,8 & 4,0 & \\
\hline Dispacidad básica & 149 & 17,2 & 6,5 & & 10,6 & 3,6 & & 6,6 & 4,4 & \\
\hline
\end{tabular}

* Desviación estándar.

Tabla 5

Distribución de las variables de control según el nivel de discapacidad

\begin{tabular}{|c|c|c|c|c|c|c|c|}
\hline \multirow{2}{*}{ Variable } & \multicolumn{2}{|c|}{ Sin discapacidad } & \multicolumn{2}{|c|}{ Discapacidad instrumental } & \multicolumn{2}{|c|}{ Discapacidad básica } & \multirow[b]{2}{*}{$\mathbf{p}$} \\
\hline & $\mathbf{n}$ & $\%$ & $\mathbf{n}$ & $\%$ & $\mathbf{n}$ & $\%$ & \\
\hline Total población & 794 & 63,8 & 290 & 23,3 & 160 & 12,9 & \\
\hline \multicolumn{8}{|l|}{ Sexo } \\
\hline Hombre & 309 & 58,2 & 160 & 30,1 & 62 & 11,7 & $<0,0005$ \\
\hline Mujer & 485 & 68,0 & 130 & 18,2 & 98 & 13,7 & \\
\hline \multicolumn{8}{|l|}{ Ingresos } \\
\hline $600 €$ o menos & 179 & 60,9 & 71 & 24,1 & 44 & 15,0 & 0,337 \\
\hline Más de $600 €$ & 610 & 64,8 & 218 & 23,2 & 113 & 12,0 & \\
\hline \multicolumn{8}{|l|}{ Vivienda } \\
\hline Propietario & 616 & 63,9 & 233 & 24,2 & 115 & 11,9 & 0,132 \\
\hline No propietario & 176 & 63,8 & 56 & 20,3 & 44 & 15,9 & \\
\hline \multicolumn{8}{|l|}{ Estudios } \\
\hline Primarios o menos & 462 & 60,6 & 196 & 25,7 & 104 & 13,6 & 0,013 \\
\hline Secundarios o más & 321 & 68,9 & 92 & 19,7 & 53 & 11,4 & \\
\hline \multicolumn{8}{|l|}{ Eventos } \\
\hline Ninguno & 71 & 70,3 & 17 & 16,8 & 13 & 12,9 & 0,157 \\
\hline 1,263 & 535 & 65,0 & 188 & 22,8 & 100 & 12,2 & \\
\hline 4 o más & 188 & 58,8 & 85 & 26,6 & 47 & 14,7 & \\
\hline Comorbilidad* & \multicolumn{2}{|c|}{$2,1(1,6)$} & \multicolumn{2}{|c|}{$2,6(1,7)$} & \multicolumn{2}{|c|}{$3,3(1,7)$} & $<0,0005$ \\
\hline Edad* & \multicolumn{2}{|c|}{$72,7(1,6)$} & \multicolumn{2}{|c|}{$72,7(1,7)$} & \multicolumn{2}{|c|}{$73,1(2,1)$} & 0,097 \\
\hline
\end{tabular}

* Para la edad y la comorbilidad se muestra la media y la desviación estándar.

para desarrollar actividades de cuidado del hogar entre los varones de esta generación (63). Por el contrario, la prevalencia de discapacidad básica es dos puntos superior entre las mujeres de este grupo de edad (13,7 versus 11,7). La prevalencia de discapacidad es también mayor entre la población sin estudios o con estudios primarios ( $p=0,013)$. De la misma forma, la media de enfermedades diagnosticadas aumenta significativamente $(\mathrm{p}<0,0005)$ a medida que se pasa de "sin discapacidad" $(2,1)$ a "discapacidad instrumental" $(2,6)$ y "discapacidad básica” $(3,3)$.
Red social y discapacidad: En la tabla 6 se especifican las Odds Ratios obtenidas al analizar, mediante regresión logística multinominal, la relación entre la red social global y desagregada en sus dos componentes y la presencia de discapacidad (instrumental o básica) frente a la ausencia de la misma. Las variables de control introducidas en el modelo fueron todas aquellas que en los análisis bivariados mostraron un grado de significación inferior a 0,25. La única variable que no cumplió este requisito fue el nivel de ingresos económicos del hogar. 
Tabla 6

Odds Ratio (OR) e intervalos de confianza al 95\% (IC95\%) del modelo de regresión logística multinominal que analiza la posibilidad de padecer una discapacidad básica o instrumental frente a no padecer ningún tipo de discapacidad

\begin{tabular}{|c|c|c|c|c|}
\hline \multirow{2}{*}{ Variable } & \multicolumn{2}{|c|}{ Discapacidad instrumental } & \multicolumn{2}{|c|}{ Discapacidad básica } \\
\hline & OR & IC95\% & OR & IB95\% \\
\hline Indicador global de red social & 0,97 & $0,94-0,99 *$ & 0,91 & $0,87-0,94 * *$ \\
\hline Edad & 1,01 & $0,92-1,10$ & 1,11 & $0,99-1,26$ \\
\hline Comorbilidad & 1,24 & $1,13-1,37^{* *}$ & 1,50 & $1,32-1,68 * *$ \\
\hline \multicolumn{5}{|l|}{ Sexo } \\
\hline Mujer & 1 & - & 1 & - \\
\hline Hombre & 2,23 & $1,64-3,04 * *$ & 1,19 & $0,77-1,82$ \\
\hline \multicolumn{5}{|l|}{ Vivienda } \\
\hline Propiedad & 1 & - & 1 & - \\
\hline No propiedad & 0,77 & $0,52-1,15$ & 0,79 & $0,48-1,30$ \\
\hline \multicolumn{5}{|l|}{ Nivel de estudios } \\
\hline Secundarios o más & 1 & - & 1 & - \\
\hline Primarios o menos & 1,38 & $1,01-1,90 *$ & 1,10 & $0,71-1,69$ \\
\hline \multicolumn{5}{|l|}{ Eventos biográficos } \\
\hline Ninguno & 1 & - & 1 & - \\
\hline 1,263 & 1,61 & $0,90-2,99$ & 0,95 & $0,45-2,01$ \\
\hline 4 o más & 2,21 & $1,14-4,26^{*}$ & 1,91 & $0,87-4,21$ \\
\hline \multicolumn{5}{|l|}{ Indicador desagregado } \\
\hline Índice de diversidad social & 1,01 & $0,97-1,05$ & 0,96 & $0,92-1,01$ \\
\hline Índice de participación social & 0,97 & $0,93-1,00^{*}$ & 0,83 & $0,79-0,87 * *$ \\
\hline
\end{tabular}

$* \mathrm{p}<0,05$

$* * \mathrm{p}<0,01$

Discapacidad instrumental: Por cada punto que aumenta el indicador de red social, la probabilidad de padecer una discapacidad instrumental, frente a no padecer ningún tipo de discapacidad, se multiplica por 0,97 (IC95\% 0,95-0,99), es decir, disminuye un $3 \%$. Este efecto parece deberse en mayor medida al índice de participación social que al índice de diversidad social que está en el límite de la significación (OR = 0,97; IC 95\% 0,93-1,00).

Por cada enfermedad diagnosticada, la probabilidad de padecer discapacidad instrumental frente a no padecer ningún tipo de discapacidad se multiplica por 1,24 (IC95\% 1,13-1,37). La probabilidad masculina de padecer una discapacidad instrumental duplica la femenina $(\mathrm{OR}=2,23$; IC95\% 1,64-3,04). La población sin estudios o con estudios primarios sufre una probabilidad 1,38 veces superior (IC95\% 1,01-
1,90) de padecer discapacidad instrumental que la población con estudios secundarios o superiores. Para los sujetos que declararon haber vivido cuatro o más eventos biográficos negativos la posibilidad de padecer discapacidad instrumental se multiplica por 2,21 (IC95\% 1,14-4,26) frente a los sujetos que no habían padecido ningún evento biográfico negativo en los últimos años.

Discapacidad básica: Por cada unidad que aumenta la puntuación en el indicador de red social, la probabilidad de padecer una discapacidad básica se multiplica por 0,91 (IC95\% 0,87-0,94) de forma estadísticamente significativa $(\mathrm{p}<0,0005)$, es decir, disminuye un $9 \%$. Al igual que para la discapacidad instrumental, también es el índice de participación social el que muestra un efecto significativo sobre la aparición de la discapacidad básica $(\mathrm{OR}=0,83$; IC 95\% 0,79-0,87). 
Entre las variables socio-demográficas básicas, solamente la comorbilidad muestra un efecto significativo sobre la discapacidad básica: por cada enfermedad diagnosticada, la probabilidad de padecer discapacidad básica se multiplica por 1,50 (IC95\% 1,32-1,68).

\section{DISCUSIÓN}

El hallazgo más importante de este estudio ha sido la sensibilidad que ha demostrado el indicador global de red social construido, a la hora de detectar la influencia de los aspectos estructurales del entorno social del individuo sobre el nivel de discapacidad en etapas iniciales de la vejez.

Los aspectos estructurales de las redes sociales han mostrado diferencias significativas según diversas variables sociodemográficas. La red social es más fuerte y amplia entre la población con mayores recursos socio-económicos, y se fortalece ante la ocurrencia de eventos biográficos negativos, debilitándose con la edad. Estas diferencias parecen deberse, fundamentalmente, a la participación social, mayor entre la población con mayores recursos socio-demográficos, y que se fortalece tras la ocurrencia de eventos biográficos negativos. Por el contrario, la diversidad social, más homogénea respecto a estas características, es la que en mayor medida se ve erosionada con la edad, debido a la mayor vulnerabilidad de sub-redes compuestas por miembros de las mismas cohortes que los sujetos objetos de estudio (hermanos, amigos) y, por tanto, sujetos al efecto de procesos de fragilidad, morbilidad y mortalidad.

No se han encontrado diferencias significativas por sexo en el indicador de red social, lo que podría indicar un efecto homogeneizador de la vejez sobre el entorno social de hombres y mujeres. La jubilación y la emancipación de los hijos hace que las tareas y la distribución de tiempos de hombres y mujeres sea más parecida, favoreciendo una convergencia de roles de género a estas edades ${ }^{12}$. Tan sólo se puede observar cierta diferencia por sexos en el índice de diversidad social, debido a la menor presencia de un vínculo de pareja entre las mujeres de estas edades, dada la sobre-mortalidad masculina a las mismas. Los recursos formativos no parecen tener un efecto por si mismos sobre las redes sociales en la vejez medidas con el indicador global, aunque sí en sentido contrario, sobre la participación y diversidad social. La participación social es mayor entre aquellos con un mayor nivel formativo, sin embargo, parece compensarse entre la población con menos recursos educativos, con una mayor diversidad de la red informal.

La prevalencia de discapacidad se acerca a la que otros autores han encontrado para el conjunto de la población española. En mediciones realizadas con anterioridad, la prevalencia de la discapacidad oscila entre un $26 \%$ y un $34 \%$ 24,57,58, cifras algo inferiores a las mostrada por la cohorte objeto de estudio (36\%). Esta diferencia puede deberse a la estructura por edades de la población, debido a que los estudios citados trabajan con el conjunto de población de 65 años o más, mientras que la cohorte estudiada se encuentra en una franja etárea de 70 a 74 años. Pero también puede haber diferencias debido a las formas de medición, ya que las actividades observadas no son exactamente las mismas en todos los casos (algunos estudios observan tan sólo dificultades en ABVD), y el grado de limitación tampoco es idéntico (algunas mediciones tan solo toman dificultades graves o incapacidad, mientras otras, como la utilizada en este estudio, utilizan cualquier grado de dificultad).

La prevalencia de discapacidad entre la población europea de 70-74 años es de un 
$24 \%$, según los datos del estudio europeo Survey of Health, Ageing and Retirement in Europe (SHARE) ${ }^{59}$. Sin embargo, según el mismo estudio, en el caso de España la prevalencia en este grupo de edad es de un $35 \%$, cifra semejante a la del presente estudio. Cotas similares se han encontrado en estudios realizados en Estados Unidos ${ }^{1,60-}$ 62. Los datos del estudio europeo SHARE sitúan a España como el país con una mayor prevalencia de discapacidad, junto con Grecia y Francia (29\% y $28 \%$ respectivamente).

Una variable de la que cabría esperar al menos cierta influencia en el desarrollo de discapacidades sería la edad, de no ser por la homogeneidad etárea de la población objeto de estudio. A pesar de ello, esta variable se encuentra en el límite de la significación estadística para la discapacidad básica $(\mathrm{OR}=1,11$; IC95\% 0,99-1,26; $\mathrm{p}=$ $0,065)$. Probablemente esta influencia sea más patente en el análisis de la incidencia de la discapacidad.

La única variable que, junto con el indicador global de red social, se asocia significativamente con ambos niveles de discapacidad es la comorbilidad. Parece lógico pensar que un número mayor de enfermedades diagnosticadas desemboque en la aparición de dificultades para la realización tanto de las AIVD como de las ABVD.

Entre las variables sociodemográficas básicas, es el nivel educativo la que ha mostrado una influencia significativa sobre la presencia de discapacidad instrumental. La población sin estudios o con estudios primarios -un $62 \%$ en estas edades- ve multiplicadas por 1,4 sus probabilidades de sufrir problemas instrumentales. El efecto de los recursos formativos sobre la autonomía en la vejez, mostrado por una amplia variedad de estu$\operatorname{dios}^{13,24,26,27,44}$, es mejor conocido que el efecto de los recursos sociales, y ha mostrado no perder su significación estadística al introducir éstos, por lo que parecen tener efectos diferenciados sobre la autonomía en la vejez.

Así mismo, el hecho de haber sufrido cuatro o más eventos biográficos negativos en los últimos años aumenta de manera importante las probabilidades de sufrir problemas instrumentales. Otros autores han demostrado la influencia de los eventos biográficos sobre el estado de salud emocional $^{63,64}$, pero no se conoce que hasta ahora se haya demostrado dicha influencia sobre el estado funcional.

El indicador de red social construido para este estudio se ha mostrado muy sensible a la hora de detectar el efecto de los aspectos estructurales de las relaciones sociales sobre el nivel de autonomía al inicio de la vejez. Las probabilidades de sufrir tanto discapacidad instrumental como básica disminuyen sensiblemente a medida que aumenta la diversidad de la red social, descenso que es más acusado en el caso de la discapacidad básica. Por cada unidad que disminuye la puntuación en el indicador de red social, la probabilidad de padecer una discapacidad instrumental aumenta un $3 \%$ y la de padecer una discapacidad básica un $9 \%$.

Estos resultados sugieren un efecto directo de la red social sobre la discapacidad, que podría sumarse a un hipotético efecto indirecto condicionando el estado de salud. En efecto, con un mismo estado de salud objetivo controlado con la variable comorbilidad, el riesgo de discapacidad aumenta a medida que disminuye la puntuación en el indicador de red social.

Sin embargo, el efecto de los diferentes componentes de la red social sobre la discapacidad no es homogéneo sino que viene determinado en mayor medida por la participación social que por la diversidad de la red social. En efecto, las personas que no tienen ninguna discapacidad han obtenido la 
misma puntuación media en el índice de diversidad social que las personas que declaran discapacidad instrumental. En cambio, para la discapacidad básica si que existe una diferencia importante en la puntuación media. Por lo que respecta a índice de participación social, las puntuaciones medias obtenidas presenten una clara tendencia decreciente según se pasa de sin discapacidad a discapacidad instrumental y a discapacidad básica, siendo esta diferencia altamente significativa. Además, las OR obtenidas por el índice de diversidad social tanto para la discapacidad instrumental como para la discapacidad básica, no presentan resultados significativos y para el índice de participación social, si que demuestra una potente influencia sobre la aparición de los dos tipos de discapacidad, en mayor medida para la discapacidad básica.

Así pues, se puede afirmar que, al igual que en otros ámbitos geográficos ${ }^{26-28,44,65}$, la diversidad de la red social tomada en su conjunto resulta predictora de discapacidad entre la población urbana española entre 70 y 74 años, así como del nivel de gravedad de la misma. Hay que añadir que este efecto se debe principalmente a la participación social más que a la red social familiar, lo que es consistente con los hallazgos de otros estudios tanto europeos ${ }^{26}$ como españoles $^{12}$ y americanos ${ }^{20}$. Cabría esperar, por tradición cultural, que la red social familiar tuviera un mayor efecto en una cultura mediterránea como la española, pero no ha resultado ser así ya que, al igual que en otros estudios españoles ${ }^{12}$, ha sido la participación social la que parece proteger en mayor medida de la aparición de la discapacidad. Una explicación a esta circunstancia la podemos encontrar en Santamarina ${ }^{66}$ que sugiere que los mayores construyen su identidad social en oposición al estereotipo presente en España que asimila la vejez con inutilidad, pasividad, dependencia y muerte social. Así, la participación en actividades sociales es una muestra de persistencia en el entramado social, a la vez que estas activi- dades tienen un valor simbólico de salud, de posibilidad de mover el propio cuerpo ${ }^{12}$.

No obstante, estos resultados hay que tomarlos con cierta precaución ya que, debido a la naturaleza transversal del estudio, no podemos confirmar la dirección de la asociación entre las variables con lo que se puede producir el fenómeno de la causalidad inversa. Pese a ello, la muestra con la que se trabaja es bastante amplia, se ha obtenido un alto grado de respuesta y se han usado instrumentos validados para las diferentes variables contempladas. Una limitación a la generalización de los resultados podría ser que se trabaja con una población muy homogénea. En efecto, es una población exclusivamente urbana y joven, con un margen de edad estrecho, entre 70 y 74 años. Sin embargo, diversos estudios han demostrado la inexistencia de diferencias en los riesgos determinantes de la salud en la vejez. En cuanto a la edad, se seleccionó esta cohorte de población en las primeras edades de riesgo de inicio de deterioro funcional y cognitivo para facilitar la evaluación de la situación de los mayores al inicio del periodo y observar la progresión del proceso.

\section{AGRADECIMIENTOS}

El proyecto "Procesos de Vulnerabilidad en la Vejez", que ha generado los datos utilizados en el presente análisis, ha sido financiada por el Ministerio de Sanidad de España a través del Fondo de Investigación Sanitaria (PI04/0552) y desarrollado por un equipo multidisciplinar perteneciente a diferentes universidades, y centros de investigación y gestión. Los componentes del equipo de investigación son: Antonio Abellán, Ana Barderas, Alicia Barrasa, Miguel Ángel Escobar, Carmen Gallardo, Raquel Hidalgo, Isabel Larrañaga, Silvia Marcu, Maria Teresa Moreno, Marilia Nicolás, Ángel Otero, Dolores Puga y Ángel Rodríguez Laso. 


\section{BIBLIOGRAFÍA}

1. Waidmann TA, Liu K. Disability Trends among Elderly Persons and Implications for the Future. J Gerontol. 2000;55B(5):298-307.

2. Abellán A, Puga D, Fundación E. La dependencia entre los mayores. CECS Informe España 2001. Madrid; 2001. p. 187-246.

3. Simons LA, McCallum J, Friedlander Y, Simons J. Healthy ageing is associated with reduced and delayed disability. Age Ageing. 2000;29:146-8.

4. Jette A, Branch L, Berlin J. Musculoskeletal Impairments and Physical Disablement Among the Aged. J Gerontol. 1990 Nov;45(6):M203-8.

5. Freedman VA, Martin LG, Schoeni RF. Recent trends in disability and functioning among older adults in the United States. JAMA. 2002; 288(24): 3137-46.

6. Subramanian SV, Kawachi I. The macro-economic determinants of health. Annu Rev Public Health 2002;23:287-302.

7. Ebrahim S. Locomotor disability in a cohort of British men: the impact of lifestyle and disease. Int J Epidemiol. 2000;29:478-86.

8. Ortún V. Desigualdad y salud. Política y Sociedad. 2000;35:65-71.

9. MacIntyre S, Ellaway A. Ecological Approaches: Rediscovering the Role of the Physical and Social Environment. Social Epidemiology: Oxford University Press; 2000. p. 332-48.

10. Dear M, Wilton R. Seeing people differently: the sociospatial construction of disability. Environment and Planning DSociety and Space. 1997; 15:455-80.

11. Link B, Phelan J. Social conditions as fundamental causes of disease. J Health Soc Behav. 1995:80-94.

12. Otero A, Zunzunegui MV, Béland F, RodríguezLaso A, García de Yébenes MJ. Relaciones sociales y envejecimiento saludable. Bilbao Fundación BBVA; 2006.

13. Otero A, Zunzunegui V, Rodríguez-Laso A, Aguilar D, Lázaro P. Volumen y tendencias de la dependencia asociada al envejecimiento en la población española. Rev Esp Salud Pública. 2004;78:201-13.

14. Grundy E, Glaser K. Socio-demographic differences in the onset and progression of disability in early old age: a longitudinal study. Age Ageing. 2000;29(2).

15. Freedman VA, Martin LG. The Role of Education in Explaining and Forecasting Trends in Functional Limitations among Older Americans. Demography. 1999;36(4):461-73.

16. Regidor E, Navarro P, Domínguez V, Rodríguez C. Inequalities in income and long term disability in Spain: an lisis of recent hypotheses using cross sectional study based on individual data. BMJ. 1997;315:1130-5.

17. Stern Y, Gurland B, Tatemichi TK, Tang MX, Wilder D, Mayeux R. Influence of education and occupation on the incidente of Alzheimer's disease. JAMA. 1994;271(13):1004-10.

18. Preston SH, Taubman P, Martin Lg PSHE. Socioeconomic Differences in Adult Mortality and Health Status. Demography of Aging. Washington: National Academy Press; 2005. p. 179-318.

19. Guralnik J, Land K, Blazer D, Fillenbaum C, Branch L. Educational status and active life expectancy among blacks and whites. N Engl J Med. 1993;329:126-7.

20. Mendes de Leon CF, Glass TA, Berkman LF. Social engagement and disability in a community population of older adults: the New Haven EPESE. Am J Epidemiol. 2003 Apr 1;157(7):63342.

21. Zunzunegui MV, Beland F, Otero A. Support from children, living arrangements, self-rated health and depressive symptoms of older people in Spain. Int J Epidemiol. 2001;30(5):1090-9.

22. Zunzunegui MV, Alvarado B, Del Ser T, Otero A. Social networks, social integration, and social engagement determine cognitive decline in community-dwelling spanish older adults. J Gerontol B Psychol Sci Soc Sci. 2003 Mar;58(2):S93-S100

23. Avlund K, Lund R, Holstein BE, Due P. Social relations as determinant of onset of disability in aging. Arch Gerontol Geriatr. 2004;38:85-99.

24. Puga D. Dependencia y necesidades asistenciales de los mayores en España. Previsión al año 2010. Alcobendas (Madrid): Fundación Pfizer; 2003.

25. Thierry X. Risques de mortalité et de surmortalité au cours des dix premières années de veuvege. Population. 1999;54(2):204.

26. Mendes de Leon C, Gold DT, Glass T, Kaplan L, George LK. Disability as a function of social net- 
works in elderly African American and Whites: The Duke EPESE 1986-1992. 1: J Gerontol B Psychol Sci Soc Sci. 2001 May;56(3):S179-90.

27. Giles LC, Metcalf PA, Glonek GF, Luszcz MA, Andrews GR. The effects of social networks on disability in older australians. J Aging Health. 2004;16(4):517-38.

28. Avlund K, Lund R, Holstein BE, Due P, SakariRantala R, Heikkinen RL. The impact of structural and functional characteristics of social relations as determinants of functional decline. 1: J Gerontol B Psychol Sci Soc Sci. 2004 Jan; 59(1): S44-51

29. Glass TA, Mendes de Leon C, Marottoli RA, Berkman LF. Population based study of social and productive activities as predictors of survival among elderly Americans. BMJ. 1999;319:47883.

30. Vogt TM, Mulloody JP, Ernst D, Pope CR, Hollis JF. Social networks as predictors of ischemic heart disease, cancer, stroke and hypertension: incidence, survival and mortality. J Clin Epidemiol. 1992;45:659-66.

31. Seeman T. Health promoting effects of friends and family on health outcomes in older adults. Am J Health Promot. 2000;14:362-70.

32. Mendes de Leon C, Glass T, Beckett LA, Seeman T, Evans DA, Berkman LF. Social networks and disability transitions across eight intervals of yearly data in the New Haven EPESE. 1: J Gerontol B Psychol Sci Soc Sci. 1999 May;54(3):S16272.

33. Verbrugge LM, Jette AM. The disablement process. Social Science \& Medicine. 1994;38:1-14.

34. Pope AM, Tarlov AR. Disability in America: Toward a national agenda for prevention. Washington DC Division of Health Promotion and Disease Prevention. Institute of Medicine; 1991.

35. Nagi SZ, Pope AM, Tarlov AR. Disability concepts revisited: Implications for prevention. Disability in America: Toward a national agenda for prevention. Washington DC: Division of Health Promotion and Disease Prevention. Institute of Medicine; 1991.

36. Nagi SZ, Sussman MB. Some conceptual issues in disability and rehabilitation. Sociology and rehabilitation. Whasington, ASA; 1965.

37. Nagi SZ. A study in the evaluation of disability and rehabilitation potential: Concepts, methods, and procedures. Am J Public Health. 1964;54 $1568-79$

38. Ministerio de Trabajo y Asuntos S, Imserso. Clasificación Internacional del Funcionamiento de la Discapacidad y de la Salud. Madrid 2001.

39. World Health O, Who. ICF: International clasification of functioning, disability and health. Geneva, Switzerland; 2002.

40. Beydoun MA, Popkin BM. The impact of socioeconomic factors on functional status dacline among community-dwelling older adults in China. Soc Sci Med. 2005;60:2045-57.

41. Puga D. La dependencia de las personas con discapacidad: entre lo sanitario y lo social, entre lo sanitario y lo público. Rev Esp Salud Pública. 2005;79(3):327-30.

42. Due P, Holstein B, Lund R, Modvig J, Avlund K. Social relations: network, support and relational strain. Soc Sci Med. 1999;48:661-73.

43. Berkman LF, Glass T. Social integration, social networks, social support and health. In: Berkman LF, Kawachi I, editors. Social epidemiology. New York: Oxford University Press; 2000. p. 137-73.

44. Unger JB, McAvay G, Bruce ML, Berkman LF, Seeman T. Variation in the impact of social network characteristics on physical functioning in elderly persons: MacArthur studies of successful aging. : J Gerontol B Psychol Sci Soc Sci. 1999 Sep;54(5):S245-51.

45. Berkman L, Melchior M, Chastang J, Niedhammer I, Leclerc A, Goldberg M. Social Integration and Mortality: A Prospective Study of French Employees of Electricity of France-gas of France. Am J Epidemiol. 2004;159(2):167-74.

46. Seeman TE, Berkman LF, Charpentier PA, Blazer DG, Albert MS, Tinetti ME. Behavioral and psychosocial predictors of physical performance: MacArthur studies of successful aging. J Gerontol A Biol Sci Med Sci. 1995 Jul;50(4):M177-83.

47. Strawbridge WJ, Cohen RD, Shema SJ, Kaplan GA. Successful aging: predictors and associated activities. Am J Epidemiol. 1996 Jul 15; 144(2): $135-41$.

48. Seeman TE, Bruce ML, McAvay GJ. Social network characteristics and onset of ADL disability: MacArthur studies of successful aging. : J Gerontol B Psychol Sci Soc Sci. 1996 Jul;51(4):S191200. 
49. Bassuk SS, Glass TA, Berkman LF. Social disengagement and incident cognitive decline in community-dwelling elderly persons. Ann Intern Med. 1999 Aug 3;131(3):165-73.

50. Katz S, Akpom CA. A measure of primary sociobiological functions. Int J Health Serv. 1976;6 (3):493-508.

51. Lawton MP, Brody EM. Assessment of older people: Self-maintaining and instrumental activities of daily living. Gerontologist. 1969;9:179-86.

52. Glass T, Mendes de Leon C, Seeman T, Berkman LF. Beyond single indicators of social network: a LISREL analysis of social ties among the elderly. Soc Sci Med. 1997;44:1503-17.

53. Hosmer DW, Lemeshow S. Applied logistic regression. New York: Willey; 1989.

54. Zabalegui A, Escobar MA, Cabrera E, Gual MP, Fortuny M, Mach G, et al. Análisis del programa educativo PECA para mejorar la calidad de vida de las personas mayores. Aten Primaria. 2006 Mar 31;37(5):260-5.

55. Sancho M, Abellán A, Puga D, Pérez L, Imserso. Informe 2004. Las personas mayores en España. Madrid; 2005.

56. (IMSERSO) Idmyss. Informe 2002. Las personas mayores en España. Madrid; 2002.

57. INE. Encuesta de Discapacidades, Deficiencias y Estado de Salud 1999. Resultados detallados. Madrid: INE; 2002.

58. Casado D, López G. Vejez, dependencia y cuidados de larga duración. Barcelona: Fundación La Caixa; 2001.
59. SHARE: Health, ageing and retirement in Europe; 2005.

60. Manton KG, Gu X. Changes in the prevalence of chronic disability in the United States black and nonblack population above age 65 from 1982 to 1999. PNAS. 2001;98(11):6354-9.

61. Crimmins E, Saito E, Reynolds SL. Futher evidence on recent trends in the prevalence and incidence of disability among older Americans from two sources: the LSOA and the NHIS. J Gerontol B Psychol Sci Soc Sci. 1997;52 59-71.

62. Schoeni RF, Freedman VA, Wallace RB. Persistent, consistent, widespread, and robust? another look at recent trends in old-age disability. J Gerontol B Psychol Sci Soc Sci. 2001;56:206-18.

63. Persson G. Life event ratings in relation to sex and marital status in a 70-year-old urban population. Acta Psychiatr Scand. 1980;62(2):112-8.

64. Fiske A, Gatz M, Pedersen NL. Depressive symptoms and aging: the effects of illness and non-health-related events. 1: J Gerontol B Psychol Sci Soc Sci. 2003 Nov;58(6):P320-8

65. Michael YL, Berkman LF, Colditz GA, Kawachi I. Live arrangements, social integration, and changes in functional health status. Am J Epidemiol. 2001;153(2):123-53.

66. Santamarina C. La percepción que los mayores tienen de si mismos. En percepciones sociales sobre las personas mayores. Madrid, Instituto de Migraciones y servicios sociales (IMSERSO); 2002. 
